\title{
Equilibrio funcional de las organizaciones basado en el poder
}

\section{Functional equilibrium of power based organizations}

págs. $135-143$
$\begin{gathered}\text { Grupo de Investigación: Competitividad empresarial } \\ \text { Línea de Investigación: Mercados financieros globalizados } \\ \text { Clemencia Martínez Aldana }\end{gathered}$

Recibido: 16 de febrero de 2016 Aceptado: 25 de abril de 2016

\section{Resumen}

El presente documento expone el pensamiento de Henry Mintzberg y de Thomas Hobbes en relación al tema de poder, como factor esencial para el equilibrio de las organizaciones. De acuerdo con Mintzberg se describen los sistemas los sistemas que intervienen en la coalición interna de las organizaciones. Según las ideas de Hobbes, se fundamenta cómo a partir de la divergencia entre el estado de naturaleza humana y el estado de la sociedad civil, debe existir un poder que obliga a cumplir acuerdos, no por simple voluntades verbales, sino por una ley que compromete.

Palabras clave: Poder, organizaciones, divergencia, sociedad civil, acuerdos.

\begin{abstract}
This document contains the thought of Henry Mintzberg and Thomas Hobbes on the issue of power as essential factor for organizational balance. In relation with Mintzberg, the establishment of systems that involves the internal coalition of organizations. As for Hobbes, it is based how from the divergence between the state of human nature and the state of civil society, there must be a power that forces enforce agreements, not only by simple verbal wills, but by a law that commits.
\end{abstract}

Keywords: power, empowerment, organizations, human nature, civil society, agreements.

- Economista, docente investigadora Fundación Universidad de América. clemencia.martinez@investigadores.uamerica.edu.co 


\section{Introducción}

Thomas Hobbes hace referencia a dos razones por las que la humanidad llega a cumplir verbalmente: una por temor a ser excluido o castigado y la otra por la satisfacción altruista de cumplir su palabra. La última asegura es poco común entre personas que buscan poder, reconocimiento, prosperidad material, y asegura es la mayoría de la humanidad. En el caso del temor, especifica que este sentimiento es percibido por quienes temen a faltarle a un ser superior (espíritu invisible) o a un humano (visible) siendo este el más común. En el caso del espiritual, está latente en la innata naturaleza humana, antes que ser parte de una sociedad civil. En segundo, el temor no es sutil, porque la condición humana natural de desigualdad en el poder, no se discierne en espíritu sino en la lucha por ganar reconocimiento y posición material y no se logra acuerdo verbal de paz ante la avaricia, la codicia la ambición u otro sentimiento negativo.

Sin embargo, la justicia alcanzada por este medio no excluye la razón en la que hombres se entregan a lograr el cuidado personal por su propia voluntad sin afectar los intereses de otro o de la colectividad. A esta posición, propone irrazonable, lograr beneficios involucrándose en el conjunto de quienes rechazan el dominio y la invasión de los derechos particulares a costa del poderío individual. Igualmente está fuera de la razón que una persona haga un algo destructible y el resultado por casualidad se torne a su propio beneficio; o que en ausencia de un poder común la(s) persona(s) apoyada(s) por otras se vuelvan en su contra. En ese orden quien rompa un pacto y lo considere razonable, no podria pertenecer a una sociedad que busca la paz.

Por otra parte incluyó fuera de la razón, alcanzar soberanía por la rebelión, porque también se incita a otros que aprendan a hacerlo por ese medio. En ese orden para Hobbes lo más relevante es la justicia definida como el cumplimiento de lo pactado y la identifica como una regla de la razón y la eleva a nivel de ley de naturaleza.

\section{Método}

Henry Mintzberg (1992, el poder en las organizaciones) en su obra El Poder en la Organización, afirma que en las organizaciones existen unos sistemas de influencia que contribuyen a la coalición interna y los clasifica en cuatro sistemas: de autoridad, de ideología, de habilidad y de política, los cuales a su juicio actúan de forma individual o conjunta según la situación específica y por lo tanto no son excluyentes entre sí.

A continuación se ilustra cómo cada uno de los sistemas propuestos por Mintzberg, contribuye al equilibrio:

1. El control personal en el sistema de autoridad, propicia el equilibrio visible en las responsabilidades pertinentes y sobrepasa la inercia latente de otros sistemas de la coalición interna. Este es el caso de una alerta del entorno que atenta a la supervivencia de la organización, ante lo cual se debe de inmediato dar giro a quien ejerce la responsabilidad concreta y puntual para retornar al equilibrio (Hamblin, 1958).

Igualmente el sistema de ideología en este escenario a juicio de Mintzberg, por sí solo no contribuye al equilibrio, porque puede estar sesgada a una creencia muy acentuada por fuera de la cual no hay autoridad ni equilibrio al interior de la organización porque se resiste al cambio.

Por otra parte, el sistema de autoridad prevaleciente en una organización llega a entorpecer su funcionamiento, cuando acciones burocráticas acentuadas empoderan a algún agente en posición a los intereses del colectivo interno e impiden el desarrollo organizacional. En el caso puntual del sistema de habilidades y política, cuando prevalecen exclusivamente, llegan a crear escenarios de discusión y retoricas que entorpecen las decisiones y empantanan la gestión. 
LINNEA DE INVESTIGACIÓN: MERCADOS FINANCIEROS GLOBALIZADOS

A juicio de Mintzberg el control personal en el sistema de autoridad es efectivo en función del equilibrio, cuando está libre de dogmas y tradiciones que concentran el poder y la toma de decisiones, lo cual impide que los agentes procedan por su propia percepción y por la intuición que les viene del libre albedrío y del pensamiento con sabiduria, y encaminan sus acciones en cumplimiento de los objetivos trazados y de los ajustes suscitados en el camino.

Al respecto es relevante citar al filósofo Thomas Hobbes, quien planteó que la autoridad es fundamental, porque sin ella no hay dirección ni control, y en ese orden, todos actúan indiscriminadamente sin objetivos específicos y coherentes, propiciando la intervención de personas inescrupulosas, fuertes de carácter y con capacidades de dominio político e ideológico.

Por su parte Mintzberg plantea que no se debe excluir a la autoridad o hacerla imprescindible y única, sino que debe existir un control personal que esté presente con los demás sistemas y se rija por un libre juego de roles y de participación coherente según la Teoría de los Nash, como estrategia para la toma de decisiones. Lógicamente es inaudito asumir que el control de un solo agente sea suficiente, porque este puede convertirse en un elemento netamente autoritario y arbitrario.

2. El control burocrático en el sistema de autoridad, facilita la estabilidad y propende por la gestión sistémica, contribuyendo a solucionar ineficiencias de los otros sistemas de influencia. El autor señala que cuando la burocracia se toma como función preponderante, conduce a que prevalezcan habilidades concentradas en poder de muchos y se dilata demasiado el poder. A su vez el poder político como representante de la burocracia cuando es exclusivo y concentrado, repele la estabilidad organizacional. Si es el caso de tomar la ideología a la luz del concepto bu- rocrático, llega a ocasionar un proselitismo que concentra demasiado el poder por la estandarización de procesos y normas que hacen la gestión cuadriculada y cerrada.

3. El sistema de ideología articulado con el sistema de autoridad y el de habilidad, se convierte en generador de vida para la estructura organizacional y repele posiciones egoístas de los otros sistemas de influencia.

Es claro que los sistemas no pueden actuar independientemente entre sí y del entorno porque el cumplimiento de los objetivos se diluye. Esta situación se observa cuando hay división entre un sistema y otro, a lo cual se suma la prevalencia de intereses oportunistas por parte de quienes lo lideran, bien sea porque tienen atributos en el desempeño de habilidades y destrezas, o por que gozan de poder de convocatoria y aglutinan grupos sociales. Estos casos no ocurren cuando la organización tiene una ideología propia y los agentes internos comulgan a conciencia con los principios, y se vinculan por propia convicción y no por caso fortuito o por sustracción de materia.

4. El sistema de habilidad se requiere para garantizar que el poder se localice donde yace la habilidad y el conocimiento de la organización y por ende no se asigna a quienes no gozan de tales atributos. En el supuesto caso de que un gran poseedor de conocimientos y experticia tenga que operar en un sistema de autoridad, posiblemente dependerá de mandos medios que no le permitirán desempeñarse libremente ni poner en juego su saber y experiencia. Si por otra parte está en un sistema de ideología, es posible que tampoco pueda actuar porque los dogmas no darán cabida a quienes no sigan los lineamientos teóricos y funcionales de la ideología.

5. El sistema de política se identifica como un factor fundamental para ajustar las falencias de los sistemas de autoridad, ideología y habilidad. 
En un sistema de autoridad en una coalición interna, usualmente quien ejerce la dirección de la organización traza los objetivos lo cual puede frenar el libre pensamiento de los demás agentes. Esta acción incluso distrae los efectos reales de un sistema de política porque priman los intereses de quien ejerce la dirección en el sistema de autoridad. En ese orden el poder no fluye hacia los intereses y necesidades de la organización, sino hacia las directrices particulares de quienes están en la cúspide de la pirámide burocrática (Pfeffer \& Salancink, 1974).

Esta posición llevó al autor a formular una inquietud, en torno a sí el sistema de autoridad no es competente al colectivo, será que lo es el sistema de política, el de habilidad, o el de ideología?

Al respecto Mintzber planteó cuatro sub-proposiciones para validar la eficiencia y funcionalidad del sistema de política:

a. El sistema de política facilita que los líderes naturales surjan por generación espontánea, por lo tanto, no es función ardua del sistema de autoridad conseguir líderes, porque estos se hacen visibles naturalmente. Siendo importante garantizar la permanencia de los más aventajados (sin posiciones egóicas), para reforzar y optimizar el liderazgo en la organización.

Aunque es factible la presencia de líderes sin relevante mística, quienes ejerzan influencia por su posición privilegiada, opacan a líderes preponderantes sometiéndolos por los niveles de jerarquía, dando lugar a la estructuración e implementación dentro del sistema de política a acciones que responden a intereses personales. También es el caso del liderazgo débil, localizado en los niveles medios que actúan desconociendo los conductos regulares y procediendo con políticas poco coherentes, algunas veces filtradas a los niveles superiores quienes desconocen los procedimientos ineficientes b. El sistema de política garantiza la participación de todos los integrantes internos de la organización y da acceso a la información disponible.

Dentro de este sistema toda la información que llega tiene valor y es considerada para analizarla y aplicarla según la pertinencia respectiva. Ello implica que los responsables manejen el compromiso y la identidad por lo que consideren justo y válido para aplicar en la formulación e implementación de la política (Allison, 1971). Para el logro utilizan diferentes estrategias, como presionar desde escenarios externos a la organización, recurrir a niveles jerárquicos de mayor autoridad, medios políticos de influencia, rivalizar con sus oponentes propiciando encuentros donde se desmitifican pensamientos, se refutan con argumentos poco doctos y respetables. En otros casos como lo planteó Strauss (1964), las dependencias no conocen el todo de la organización, sino que tienen una visión parcial, y al darse la interrelación entre estas, se logra un conocimiento más general que alimenta la razón de ser de la organización, el cumplimiento de los objetivos, la solución de las necesidades y por efectos de la discusión y la verbalización de las opiniones, las ideas y los temas se hacen visibles, excluyendo incluso la intervención externa como árbitro de las relaciones y se torna objetiva la interrelación ideológica. La argumentación ideológica y práctica facilita la formulación de políticas cuando va direccionada al cumplimiento de los objetivos misionales de la organización y no a los intereses individuales.

c. El sistema de política facilita la dialéctica en la organización, siempre que esté acorde con la filosofía institucional, y permite desbloquear los rezagos de los sistemas de ideología, habilidad y autoridad.

McCall (1979) plantea que cuando hay poder, este debe cambiar con los nuevos requerimientos en las organizaciones, porque de lo contrario fenecerán al no adaptarse a 
LIINEA DE INVESTIGACIÓN: MERCADOS FINANCIEROS GLOBALIZADOS

las nuevas circunstancias y tener presente que los iniciadores o fundadores de una organización, de una política o de una ideología, no son perennes en el poder (Mintzber, 1978).

Adicionalmente las personas envejecen, pierden vigencia y se aferran al poder. Como lo señalan Salancik \& Pfeffer (1977), una de las razones por las que la autoridad se suele aferrar al cambio emana de los sistemas en que operan las instituciones, al centralizar el poder bajo esquemas incoherentes con la realidad y el contexto. Es el caso de los sistemas basados en una ideología con tintes dogmáticos y normativos que conllevan a generar temor e inseguridad por nuevos planteamientos, a partir de acciones que inculcan sentimientos de culpa y castigo.

Mintzber (1979a) alude que las organizaciones pueden ser reacias al cambio cuando funcionan por patrones estandarizados (habilidades), como en el caso de las universidades y las empresas de contabilidad entre otras, que mantienen concentrado el poder entre quienes trabajan por los ideales de su profesión y le sirven con mayor empeño a esta que a la misma organización.

Cuando se presentan estos comportamientos entra a actuar el sistema de política, estructurando procesos, planes y programas que modifiquen. Ideologías, habilidades, y formas de autoridad.

d. El sistema de política facilita los procesos para la ejecución de las decisiones. Para ello se requiere que los niveles directivos previo a hacer público un plan, deben plantear estrategias, y conformar alianzas, que garanticen el logro de los objetivos trazados, es decir deben ganarse la voluntad política de la organización.

De lo enunciado en los numerales 1 a 4 , se infiere a juicio de los autores citados que el sistema de política por sí solo no es siempre fun- cional, es decir racional frente a las necesidades de la respectiva organización, como tampoco lo son los demás sistemas (ideología, habilidad y autoridad).

Por lo tanto es importante analizar cómo pueden ser coherentes y pertinentes estos sistemas de influencia operando individual o conjuntamente para lograr el equilibrio al interior de una organización, y en un momento dado corregir los errores causados por el dominio de uno de los sistemas. Siendo un hecho común que en las coaliciones internas un sistema específico de influencia sea dominante.

Por lo anterior Mintzberg describe cinco formas en que domina un sistema generando coaliciones internas:

- Coalición interna personalizada: domina el control personal (sistema de autoridad), hay una fuerte concentración de poder en la cima de la organización. No se vislumbra acción del sistema de política, no se requiere habilidad porque no se delega en nadie, no hay lugar a niveles burocráticos porque estos aminoran el poder centralizado. Es factible algún grado de injerencia del sistema ideológico cuando este se le subordina al control personal apoyando al líder.

- Coalición interna burocrática: dominan los controles burocráticos (sistema de autoridad), es decir, el poder se ejerce mediante la estandarización de los procesos laborales y productivos en los niveles inferiores de la pirámide. No permite niveles de habilidad porque se salen de los parámetros de estandarización y ponen en peligro las formas de control.

- Coalición interna ideológica: domina el sistema de ideología. Se da una integración de los agentes quienes internalizan los objetivos y necesidades de la organización. La socialización, adoctrinamiento o identidad con la filosofía y los ideales de la organización, hacen de ésta la coalición interna más igua- 
litaria y requiere de los menores niveles de control. No hay espacio para la influencia política ni para la habilidad, porque existe una homogeneidad ideológica donde no cabe el profesionalismo. Se requiere de un fuerte liderazgo carismático

- Coalición interna profesional: domina el sistema de habilidad. El poder está centrado en el conocimiento complejo, por lo que se requiere contratar personas muy expertas, luego no hay espacio para el dominio de fuertes ideologías o de relevantes niveles de autoridad. Hay cabida para la política porque esta lleva implícito el cambio, siendo aquel un factor dinámico del conocimiento, al igual que la habilidad, porque es fundamento del cambio de la evolución técnica y científica.

- Coalición interna politizada: domina el sistema de política. En este escenario no hay foco de poder, porque las estrategias grupales y gremiales conllevan a la fluidez de alianzas y a la negociación de la interdependencia.

Una vez expuesto el pensamiento de Mintzberg, se presenta la postura de Thomas Hobbes, cuando planteó que la autoridad es fundamental porque sin ella no hay dirección, ni control, y en ese orden todos actúan indiscriminadamente, sin objetivos específicos y coherentes, propiciando la intervención de personas inescrupulosas, fuertes de carácter y con capacidades de dominio político e ideológico.

Hobbes criticó el postulado de los griegos que afirmaban la disposición natural imnata del hombre para vivir en sociedad, y que con el hecho de llegar a acuerdos sobre determinadas condiciones, a los que denominaron leyes, era suficiente fundamentación para estructurar una teoría política.

Este axioma a su juicio no fue muy contundente, porque resultó de un diagnóstico muy superficial de la naturaleza humana, requiriéndose de un fuerte trabajo racional, partiendo del ser humano con un carácter condicionado a reac- cionar y colaborar cuando existe de por medio el temor a un poder común, que lleve a la mutua desconfianza y al temor entre sí. Hobbes a partir de esta argumentación explicó en detalle la inflexión entre el estado de naturaleza y el estado político de la sociedad civil. En el primero existe la igualdad natural de todos los hombres, por convivir en completa libertad, y en el segundo funcionan bajo el poder de un Estado con leyes que someten a todas las personas en función de una convivencia civilizada.

La libertad es un derecho natural que cada hombre tiene de usar su propio poder como desee para lograr objetivos según su juicio y razón, sin impedimentos externos de ningún tipo. Esa ley natural es una regla de la razón y lleva a diferenciar entre los conceptos de derecho y ley, entendiéndose por derecho la libertad de hacer o no hacer; y por ley, la que ata ese hacer o no hacer.

Considerando que por naturaleza el hombre tiende a vivir bajo el principio de hacer que primen exclusivamente sus propios derechos, es decir todos contra todos donde cada cual es gobernado por su exclusiva razón, llevó según Hobbes a que el hombre por preservar los logros alcanzados y hasta la propia vida, busque la paz como regla prioritaria de la razón, y en segunda instancia para que mantenga ese derecho natural de la paz, instituyó que por todos los medios a su alcance debería lograrla y hacerla posible.

Hobbes concibió los derechos como una renuncia o transferencia de perder la primacia en favor de quien reciba los beneficios. Renuncia, cuando es indiferente en quien recaigan los consiguientes beneficios. Transferencia, cuando estos benéficos se asignan a una persona determinada. Se infiere que el dejar los beneficios a otro, la persona está obligada a no intervenir de forma alguna en beneficio propio, siendo su deber valorar esa acción propia y voluntaria. Por lo tanto esa renuncia o transferencia del derecho a otro debe tener una reciprocidad consignada en un contrato, el cual puede ser 
LIINEA DE INVESTIGACIÓN: MERCADOS FINANCIEROS GLOBALIZADOS

de estricto contado y se le califica como un convenio; o puede ser pactado a futuro y se denomina convenio, es decir, se acuerda hoy y se hace efectivo a futuro.

También existe la figura de transferir beneficios sin que la acción sea mutua, de tal manera que una de las partes toma la decisión de cederlos plenamente, en este caso no hay contrato sino una donación. En cualquiera de las modalidades descritas existe un cambio de derecho.

Existe la modalidad de ofrecer a futuro por merecimiento, caso en el cual transfiere un derecho a quien, por ejemplo, venza en una lucha.

Otra forma de transferir un derecho se da cuando las partes hacen un pacto verbal de confianza mutua a futuro. Este caso es complejo por la misma naturaleza humana que cada cual busca beneficio personal desconociendo los derechos de los demás, donde todas las personas son iguales y juzgan bajo las mismas condiciones sus propia vulnerabilidad, de forma que existe en el trasfondo la inseguridad de que el otro cumpla su pacto tal como se había acordado inicialmente de palabra, porque la fuerza del verbo es débil para hacer frente a la ambición, la avaricia, el egoísmo y la envidia. Ese comportamiento de desconfianza es propio de una sociedad sin poder coercitivo el cual obliga a cumplir los pactos por temor a la sanción cuando se falta a los acuerdos, y en consecuencia obliga a no invalidar, vulnerar o irrespetar los derechos de los otros.

En una sociedad civil donde existe un poder establecido que obliga a cumplir acuerdos, se elimina el temor a que otros falten, de forma que las personas actúan no por un simple voto de acuerdo de voluntades verbales, sino por una ley que las compromete (Hobbes, 2011).

En ese orden se infiere que quienes conviven fuera de la sociedad civil hacen cuanto quieran libremente, luego se usurpan los derechos unos a otros y están en igualdad de condiciones de sentirse afectados por lo que hicieron a los demás. En cambio, quienes viven a la luz de la sociedad civil, es decir, bajo el gobierno de un estado bien concebido, saben que sus derechos van hasta donde empiezan los de los otros y tienen, en consecuencia libertad para hacer lo que quieran, respetando la ley que ampara el uso los derechos particulares de las personas.

Lo más importante es la paz y la defensa por igual que protege a todos los conciudadanos del Estado, operando bajo la razón, la paz, la seguridad y la tranquilidad. En cambio vivir sin un estado que los proteja hace que las personas cuando sientan vulnerados sus derechos, queden a merced de defenderse exclusivamente con sus propias fuerzas sin otra alternativa, por no poder acceder a un Estado instituido que apelando a leyes haga respetar los derechos (Hobbes, 1965).

Consideró relevante tener un pacto social generado por el Estado, identificado como una unidad compuesta por muchos hombres, cuya voluntad se mantiene por la voluntad de todos, de forma que está persona pública pueda hacer uso de todos los medios e instrumentos para ser equipo de presión cuando se vulnere la paz pública y la defensa social (Hobbes, loc. Cit, 14).

La libertad que toda persona tiene de usar sus propios atributos para la conservación y desarrollo de su naturaleza, es la misma para personas fuertes y débiles, luego no existe posibilidad que el hombre más grande llegue a reclamar por cualquier medio más derechos que otro, dada la igualdad a nivel de las facultades de cuerpo y espíritu a la luz del Derecho de la naturaleza humana. Lo anterior en razón a que la naturaleza de los hombres desconoce el hecho de que existen personas con mayores atributos, difícilmente creen y toman conciencia que existan personas más sabias, y ven la propia inteligencia a la mano, mientras la de otros goza de prudente distancia. Esta característica tan común en el género humano, denota la desigualdad en la humanidad. 
Hobbes enfocó su pensamiento en el gobierno, pero de forma pertinente su postura se puede aplicar a las organizaciones. Este autor considera que en la autoridad convergen todas las probabilidades, que los individuos responden a la autoridad con la esperanza de que todos hagan lo mismo. En ese orden se infiere que la autoridad fusiona a los diferentes componentes de la organización y sustenta en sí a la organización.

\section{Comentarios}

Mintzber aportó a la administración una visión de cómo los sistemas de influencia: autoridad, ideología, habilidad y política, contribuyen a la coalición interna de las organizaciones. Explicó cada uno de los sistemas y los interrelacionó, detallando que la primacía de uno u otro sobre los demás en circunstancias específicas llevan al desequilibrio interno de las organizaciones y distorsiona el sentido real del poder como fuente y factor de equilibrio.

Por otra parte Thomas Hobbes, de una forma más indirecta que la de Mintzberg, contribuyó a la administración, porque fija una estrategia desde lo institucional y plantea que debe existir un estamento supremo que regule, controle y maneje el poder, para que se interrumpa la inflexión entre el estado de naturaleza humano y el estado de la sociedad civil, al existir un poder que obliga a cumplir acuerdos, para eliminar los temores a que otros falten, haciendo que las personas actúen no por un simple voto de acuerdo de voluntades verbales, sino por una ley que los compromete.

Desde una óptica personal, logró Mintzberg esclarecer y desmitificar el concepto de poder, enmarcado como presión y dominio dentro del lenguaje común, sacándolo de ese arquetipo y llevándolo a ser considerado como una variable cualitativa que favorece procesos de gestión.

Y mi visión hacia Hobbes, es que gracias a su Leviatán las personas en las organizaciones actúan en función de la justicia, desarrollando la adaptación hacia la sociabilidad con la colectividad en cualquier posición y rol que le haya sido asignado, con equidad, cumpliendo los compromisos adquiridos en los acuerdos pactados.

Una vez expuesto el pensamiento de Minztberg y de Hobbes, infiero que la acción conjunta de todos los sistemas integrantes del poder se presenta cuando es necesario concentrar o dispersar ese poder, porque se está buscando un beneficio para la organización, debido a que hay fuerzas que lo concentran hacia el centro o por el contrario lo dispersan hacia la periferia. Este pensamiento se percibe en cualquier dimensión piramidal de una organización humana, dado que son los agentes humanos quienes la ejercen y entre los anti valores predominantes está el poder como factor de dominio y prevalencia social, retomando la frase de "poder engendra más poder", lo cual propicia la concentración del mismo y el aprovechamiento de posiciones privilegiadas.

Considero que al desarticular el poder del centro y difuminarlo, se logra en casos puntuales asignarlo a quienes realmente tienen las habilidades específicas y con mayor facilidad potencializarán la gestión pertinente. Esto explica el por qué las organizaciones deben estar en plena dialéctica de concentrar y dispersar el poder según lo requieran las circunstancias y el entorno, para que sea más eficiente la gestión en concordancia con las necesidades de la organización. Se hace reiterativo que las acciones centrípeta y centrifuga deben estar en permanente monitoreo para ajustarlas a los contextos que se requiera, e implica que los diferentes sistemas estén articulados en cuanto al direccionamiento de los mismos objetivos, la solución de las distintas necesidades de la organización, la corrección de deficiencias de cada uno de los sistemas y la obtención de resultados de mayor alcance con pleno equilibrio funcional de la organización. 
LÍNEA DE INVESTIGACIÓN: MERCADOS FINANCIEROS GLOBALIZADOS

\section{Referencias}

Allison, G.T., (1971): Esscense of Decision Explaining the Cuban Missile Crisis. Boston: Little

Brown, 1971. Copyright (@ 1971 by Graham T. Allison. Reprinted by permission of the Publisher. En H. Mintzberg. (Ed.), El Poder en la Organización. Barcelona: Ariel S.A.

Hamblin, R. L., (1958): Leadership and Crises Sociometry. En H. Mintzberg. (Ed.), El Poder en la Organización. Barcelona: Ariel S.A.

Hobbes, Th., (1965): Antología de textos políticos. Del Ciudadano. Leviatán. Selección e introducción de E. Tierno Galván. Editorial Tecnos, S.A., Madrid.

Hobbes, Th., (2011): Leviatán. Traducción Antonio Escohotado. Editorial Losada, Buenos Aires.

Hobbes, Th., 1940: Leviatán o la materia, forma y poder de una república, eclesiástica y civil. Traducción y prefacio de M. Sánchez Sarto. FCE. México.

McCall. M.W., Jr., (1979): Power, Authority and Influence", in Organizational Behavior, ed, S. Kerr. Colmbus Ohio.

Mintzberg, H. (1978). Beyond Implementation: An Analysis of the Resistance to Police Analysis, in Operational Research '78, ed. K.B. Haley. Amnsterdam, Netherlands: North Holland. 1979b.

Mintzber, (1979a) The Structuring of Organizations: A Synthesis of the Research. Englewood Cliffs: Prentice-Hall, 1979a.

Mintzberg, H. (1992). El Poder en la Organización. Barcelona: Editorial Ariel S.A.

Pfeffer, J., \& G,R.,Salancik,(1974): Organizational Decision Making as a Political Process the Case of a University Budget Administrative Science Quarterly. En H. Mintzberg. (Ed.), El Poder en la Organización. Barcelona: Ariel S.A

Salancik, J., R., \& Pfeffer, J., (1977): Who Gets Power and How the Hold on to It: A Strategic-Contingency Model of Power" Organizational Dynamic, Winter 1977. En H. Mintzberg. (Ed.), El Poder en la Organización. Barcelona: Ariel S.A

Strauss, G., (1964): Workflow Frictions, Interfunctional Rivarly, and Proffesionalism: A Case, Study of Purchansing Agents, Human Organization. En H. Mintzberg. (Ed.), El Poder en la Organización. Barcelona: Ariel S.A. 